\title{
A colonisation-inhibition culture consisting of Salmonella Enteritidis and Typhimurium $\Delta$ hilAssrAfliG strains protects against infection by strains of both serotypes in broilers
}

\author{
W. De Cort, D. Mot, F. Haesebrouck, R. Ducatelle, F. Van Immerseel* \\ Department of Pathology, Bacteriology and Avian Diseases, Faculty of Veterinary Medicine, Ghent University, Salisburylaan 133, B-9820 Merelbeke, Belgium
}

\section{A R T I C L E I N F O}

\section{Article history:}

Received 14 April 2014

Received in revised form 22 May 2014

Accepted 13 June 2014

Available online 25 June 2014

\section{Keywords:}

Salmonella

Poultry

Colonisation-inhibition

\begin{abstract}
A B S T R A C T
Consumption of contaminated poultry meat is still an important cause of Salmonella infections in humans and there is a need for control methods that protect broilers from day-of-hatch until slaughter age against infection with Salmonella. Colonisation-inhibition, a concept in which a live Salmonella strain is orally administered to day-old chickens and protects against subsequent challenge, can potentially be used as control method. In this study, the efficacy of a Salmonella Typhimurium $\Delta$ hilAssrAfliG strain as a colonisation-inhibition strain for protection of broilers against Salmonella Typhimurium was evaluated. Administration of a Salmonella Typhimurium $\Delta$ hilAssrAfliG strain to day-old broiler chickens decreased faecal shedding and strongly reduced caecal and internal organ colonisation of a Salmonella Typhimurium challenge strain administered one day later using a seeder bird model. In addition, it was verified whether a colonisation-inhibition culture could be developed that protects against both Salmonella Enteritidis and Typhimurium. Therefore, the Salmonella Typhimurium $\Delta$ hilAssrAfliG strain was orally administered simultaneously with a Salmonella Enteritidis $\Delta$ hilAssrAfliG strain to day-old broiler chickens, which resulted in a decreased caecal and internal organ colonisation for both a Salmonella Enteritidis and a Salmonella Typhimurium challenge strain short after hatching, using a seeder bird model. The combined culture was not protective against Salmonella Paratyphi B varietas Java challenge, indicating serotypespecific protection mechanisms. The data suggest that colonisation-inhibition can potentially be used as a versatile control method to protect poultry against several Salmonella serotypes.
\end{abstract}

(c) 2014 Elsevier Ltd. All rights reserved.

\section{Introduction}

Despite the implementation of numerous monitoring and control measures in broiler production, Salmonella is still an important cause of poultry meat associated human infections [1]. Broilers often become infected with Salmonella early after hatching as they are highly susceptible to infection during these first days of life [2]. This is mainly due to the absence of normal gut microbiota in young chickens and the immaturity of their immune system [3-7]. Infection during this period, even with low numbers of Salmonella, can lead to persistent carriers [8,9]. These broilers are often still infected at slaughter age, which may result in introduction of Salmonella in the slaughter house and food chain [10]. Consequently, prevention of infection during this period in which the chick is highly susceptible to infection could strongly reduce the introduction of Salmonella in the food chain.

\footnotetext{
* Corresponding author. Tel.: +32 926474 47; fax: +32 92647789

E-mail address: filip.vanimmerseel@ugent.be (F. Van Immerseel).
}

Colonisation-inhibition $(\mathrm{CI})$ is a phenomenon in which chickens are administered a live Salmonella strain that protects against subsequent challenge with another Salmonella strain [11]. By administering a $\mathrm{CI}$ strain that colonises the gut rapidly and extensively, it is possible to increase resistance to Salmonella strains quickly after hatching [12]. This concept has been recognised for a long time, and a great deal of effort has been put in developing strains that are appropriate for use as CI strains [13,14]. Earlier research demonstrated that deletion of the hilA, ssrA and fliG genes in a Salmonella Enteritidis strain resulted in a CI strain that was safe and effective in protecting broilers against challenge with a Salmonella Enteritidis wild-type strain [15]. Because there is greater inhibition within a serovar than between serovars [16], the Salmonella Enteritidis $\Delta$ hilAssrAfliG strain can be expected to mainly protect against Salmonella Enteritidis infection, and not or to a lesser extent against e.g., Salmonella Typhimurium infection. In 2011, 0.3\% of all broiler flocks were positive for Salmonella Enteritidis and Salmonella Typhimurium in Europe, while 3\% were positive for other Salmonella serotypes [1]. Consequently, if CI strains are needed that protect against these other serovars, new 
CI strains need to be developed. It is however unknown whether introduction of the hilA, ssrA and fliG mutations in a Salmonella strain belonging to another serovar yields a $\mathrm{CI}$ strain displaying the same degree of attenuation and similar protective properties as the Salmonella Enteritidis $\Delta$ hilAssrAfliG strain. Additionally, it is not known whether a combination of two or more CI strains, belonging to different serovars, is able to protect against infection by different Salmonella serovars.

In the present study, the efficacy of a Salmonella Typhimurium hilAssrAfliG deletion mutant as a CI strain was evaluated. Secondly, the protective effect of a CI culture consisting of both a Salmonella Enteritidis and Typhimurium hilAssrAfliG deletion mutant against Salmonella Enteritidis, Typhimurium and Paratyphi B var. Java infection was evaluated.

\section{Materials and methods}

\subsection{Chickens}

One-day-old Ross 308 broiler chickens were obtained from a local hatchery and housed in separate rooms in containers on wood shavings. Commercial feed and drinking water were provided ad libitum. Experiments were performed with the permission of the Ethical Committee of the Faculty of Veterinary Medicine, Ghent University, Belgium (experiment authorisation number: EC2012/96).

\subsection{Bacterial strains and deletion mutants}

A spontaneous nalidixic acid-resistant mutant of Salmonella Typhimurium strain 112910a, originally isolated from a pig stool sample [17], was used for the production of isogenic mutants. This resistance has previously been shown to have no impact on the in vivo results [13]. Deletion of hilA, ssrA and fliG genes in this strain was done using the one-step inactivation method described by Datsenko and Wanner [18]. Salmonella Typhimurium MB2136, a streptomycin resistant wild-type strain originally isolated from swine was used as a challenge strain. A nalidixic acid-resistant Salmonella Enteritidis hilAssrAfliG deletion mutant, which has been described earlier [15], was also used in this study. The original Salmonella Enteritidis 76 Sa88 strain, from which the Salmonella Enteritidis hilAssrAfliG deletion mutant is derived, was originally isolated from a poultry farm [2,19]. Salmonella Enteritidis 147 (streptomycin resistant), a strain originally isolated from chicken egg white and which is known to colonise the gut and internal organs of chickens to a high level [12,20,21], was used as a challenge strain. Additionally, a wild-type Salmonella Paratyphi B. var. Java strain (carbenicillin resistant) originally isolated from poultry, was also used as a challenge strain.

\section{Experimental design}

\subsection{Experiment 1: efficacy of a Salmonella Typhimurium hilAssrAfliG deletion mutant against experimental Salmonella Typhimurium infection}

In order to evaluate the persistence of a Salmonella Typhimurium $\Delta$ hilAssrAfliG strain in chickens and its efficacy against colonisation by a wild type Salmonella Typhimurium strain, 225 one-day-old chicks were divided into three groups of 75 animals and each group was housed in a container of $2 \mathrm{~m}^{2}$. Two groups (Group V and C) were given $10^{9} \mathrm{CFU}$ of the Salmonella Typhimurium $\Delta$ hilAssrAfliG strain by oral gavage while the third group was given sterile HBSS (Hank's Balanced Salt Solution, Invitrogen, Paisley, England) as a control (Group I). Twenty-four hours later, 25 randomly selected chickens in Group I and Group C were given $10^{5}$ CFU Salmonella Typhimurium MB2136 by oral gavage. These seeder birds were housed together with the other chickens of their group. Bacterial counts in caecum and spleen were determined for one third of the original number of chickens by bacteriological analysis at 7,21 and 42 days old. At each time point, one in three sampled animals were seeder birds. Shedding of both strains was monitored by cloacal swabbing on days $2,3,9$, $16,23,30$ and 37 .

\subsection{Experiment 2: efficacy of simultaneous administration of a Salmonella Typhimurium hilAssrAfliG and a Salmonella Enteritidis hilAssrAfliG deletion mutant against infection with several Salmonella serotypes}

In order to evaluate the efficacy of a CI culture containing a Salmonella Enteritidis $\Delta$ hilAssrAfliG and Salmonella Typhimurium $\triangle$ hilAssrAfliG strain against infection by several Salmonella serotypes, 60 one-day-old chickens were divided into 6 groups of 10 animals. Three of these groups (Group VSE, VST and VSJ) were given $0.5 \mathrm{ml}$ of a mixture containing $2 \times 10^{8} \mathrm{CFU} / \mathrm{ml}$ of the Salmonella Enteritidis $\triangle$ hilAssrAfliG strain and $2 \times 10^{8} \mathrm{CFU} / \mathrm{ml}$ of the Salmonella Typhimurium $\triangle$ hilAssrAfliG strain by oral gavage. The three remaining groups (CSE, CST and CSJ) were given sterile HBSS as a control. Twenty-four hours later, 2 randomly selected chickens in each group were given $10^{5} \mathrm{CFU}$ of a challenge strain by oral gavage. These seeder birds were then housed together again with the other chickens of their group. Groups VSE and CSE were challenged with Salmonella Enteritidis strain 147, Groups VST and CST with Salmonella Typhimurium strain MB2136 and Groups VSJ and CSJ with the wild-type Salmonella Paratyphi B var. Java strain. Bacterial counts of $\mathrm{CI}$ strains and challenge strains in caecum and spleen were determined by bacteriological analysis on day 7 .

\subsection{Bacteriological analysis}

Cloacal swabs were directly inoculated on Xylose Lysine Deoxycholate agar (XLD, Oxoid, Basingstoke, England) plates with $20 \mu \mathrm{g} / \mathrm{ml}$ nalidixic acid (Sigma-Aldrich, St. Louis, MO, USA) or $100 \mu \mathrm{g} / \mathrm{ml}$ streptomycin (Sigma-Aldrich, St. Louis, MO, USA). Samples negative after direct inoculation were pre-enriched in buffered peptone water (BPW, Oxoid, Basingstoke, England) and incubated overnight at $37^{\circ} \mathrm{C}$. One $\mathrm{ml}$ of this suspension was further enriched by adding $9 \mathrm{ml}$ tetrathionate-brilliant green broth (Merck, Darmstadt, Germany). After overnight incubation at $37^{\circ} \mathrm{C}$, this suspension was plated on XLD plates supplemented with the appropriate antibiotic. Samples of caecum and spleen were homogenised in BPW and 10-fold dilutions were made in HBSS. Six droplets of $20 \mu \mathrm{l}$ of each dilution were plated on XLD plates supplemented with $20 \mu \mathrm{g} / \mathrm{ml}$ nalidixic acid, $100 \mu \mathrm{g} / \mathrm{ml}$ streptomycin or $100 \mu \mathrm{g} / \mathrm{ml}$ carbenicillin (Sigma-Aldrich, St. Louis, MO, USA). After overnight incubation at $37^{\circ} \mathrm{C}$, the number of $\mathrm{CFU} / \mathrm{g}$ tissue was determined by counting the number of bacterial colonies on the plates.

\subsection{Statistical analysis}

GraphPad Prism 5 software was used for statistical analysis. A Fisher's exact test (one-sided) was used to analyse differences in mortality between groups. A Kruskal-Wallis test (one-way ANOVA) was used to determine statistical differences of the number of Salmonella positive cloaca swabs among groups. Bacterial counts in caecum and spleen were converted into logarithmic form for statistical analysis. Samples of caecum and spleen negative after direct plating were rated as $\log _{10}=0$. The mean $\mathrm{CFU} / \mathrm{g}$ tissue was calculated for each group on every time point and differences 
Table 1

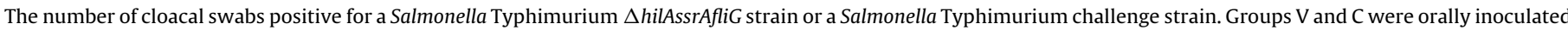

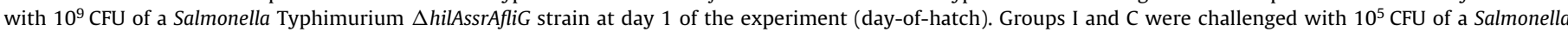
Typhimurium challenge strain on day 2 of the experiment. Samples were taken at days 2, 3, 9, 16, 23, 30 and 37 of the experiment.

\begin{tabular}{|c|c|c|c|c|c|c|c|c|}
\hline Strain & Group & Day 2 & Day 3 & Day 9 & Day 16 & Day 23 & Day 30 & Day 37 \\
\hline Salmonella Typhimurium & V & $75 / 75^{\mathrm{a}}\left(75^{\mathrm{b}}\right)$ & $75 / 75\left(72^{*}\right)$ & $49 / 49$ (47) & $47 / 47\left(30^{*}\right)$ & $24 / 24\left(20^{*}\right)$ & $24^{*} / 24\left(20^{*}\right)$ & $22^{*} / 23\left(18^{*}\right)$ \\
\hline$\Delta$ hilAssrAfliG strain & C & $75 / 75(67)$ & $\mathrm{NA} / 75\left(17^{*}\right)$ & $\mathrm{NA} / 50$ (NA) & $\mathrm{NA} / 48\left(10^{*}\right)$ & $0 / 23\left(0^{*}\right)$ & $0^{*} / 22\left(0^{*}\right)$ & $0^{*} / 22\left(0^{*}\right)$ \\
\hline Salmonella Typhimurium & I & NA & $18 / 75(8)$ & $45^{*} / 50\left(41^{*}\right)$ & $35^{*} / 49(12)$ & $5 / 25(5)$ & $10 / 25(7)$ & $10 / 25(7)$ \\
\hline challenge strain & $\mathrm{C}$ & NA & $12 / 75(0)$ & $10^{*} / 50\left(0^{*}\right)$ & $0^{*} / 48(0)$ & $0 / 23(0)$ & $0 / 22(0)$ & $0 / 22(0)$ \\
\hline
\end{tabular}

a Number of positive samples after enrichment/total number of samples.

b Number of positive samples after direct plating.

* Significant difference in positive samples between both groups ( $p$-value $<0.05)$. NA = Not available.

between groups were analysed using a Kruskal-Wallis test (oneway ANOVA) or a Mann-Whitney test. Differences with $p$-values lower than 0.05 were considered to be significant.

\section{Results}

4.1. Experiment 1: efficacy of a Salmonella Typhimurium hilAssrAfliG deletion mutant against experimental Salmonella Typhimurium infection

During the experiment, four chickens died in Group V, five in Group I and four in Group C. The observed differences in mortality were not statistically significant.

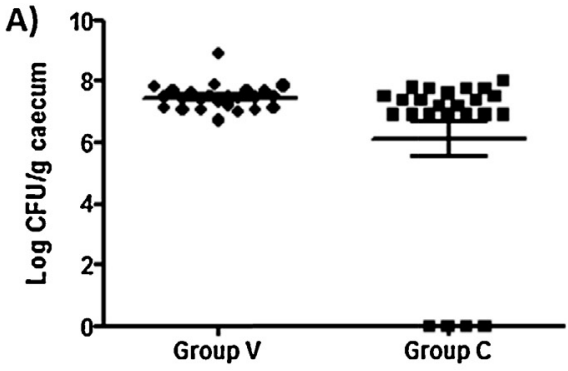

B)
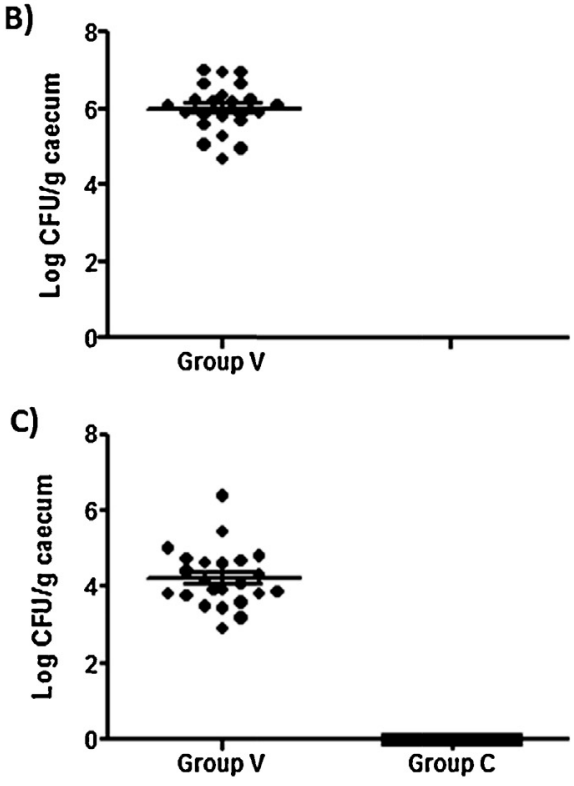

Shedding of the Salmonella Typhimurium $\Delta$ hilAssrAfliG strain remained high during the entire experiment in Group V (Table 1). Shedding of this strain decreased quickly in Group C. The Salmonella Typhimurium $\Delta$ hilAssrAfliG strain was excreted until day 16 by a limited number of chickens, after which it could no longer be detected. Shedding of the Salmonella Typhimurium challenge strain was lower in the group treated with a Salmonella Typhimurium $\Delta$ hilAssrAfliG strain during the entire experiment when compared to the sham-treated control. Statistical differences are shown in Table 1. Data on shedding of the Salmonella Typhimurium $\Delta$ hilAssrAfliG strain on days 3, 9 and 16 of the experiment are not available due to overgrowth of other bacteria on the culture media.

Bacteriological analysis of the caecum samples showed that the Salmonella Typhimurium $\Delta$ hilAssrAfliG strain colonised the caecum
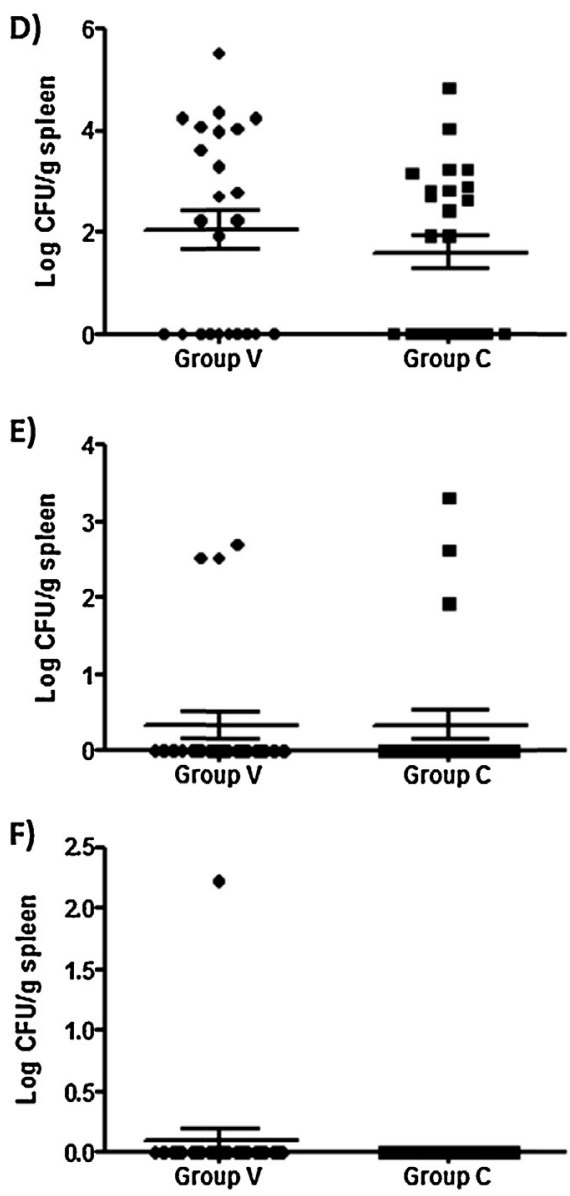

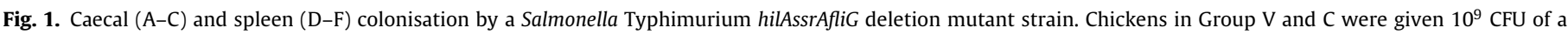

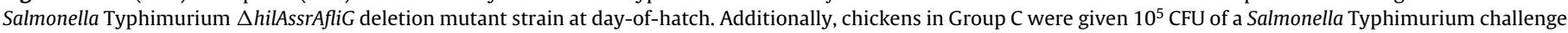

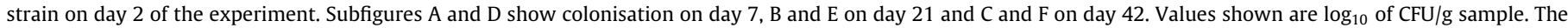

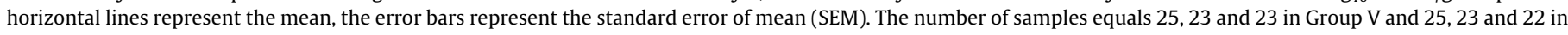
Group C on day 7, 21 and 42 respectively. No data are available for the caeca of Group C on day 21. 

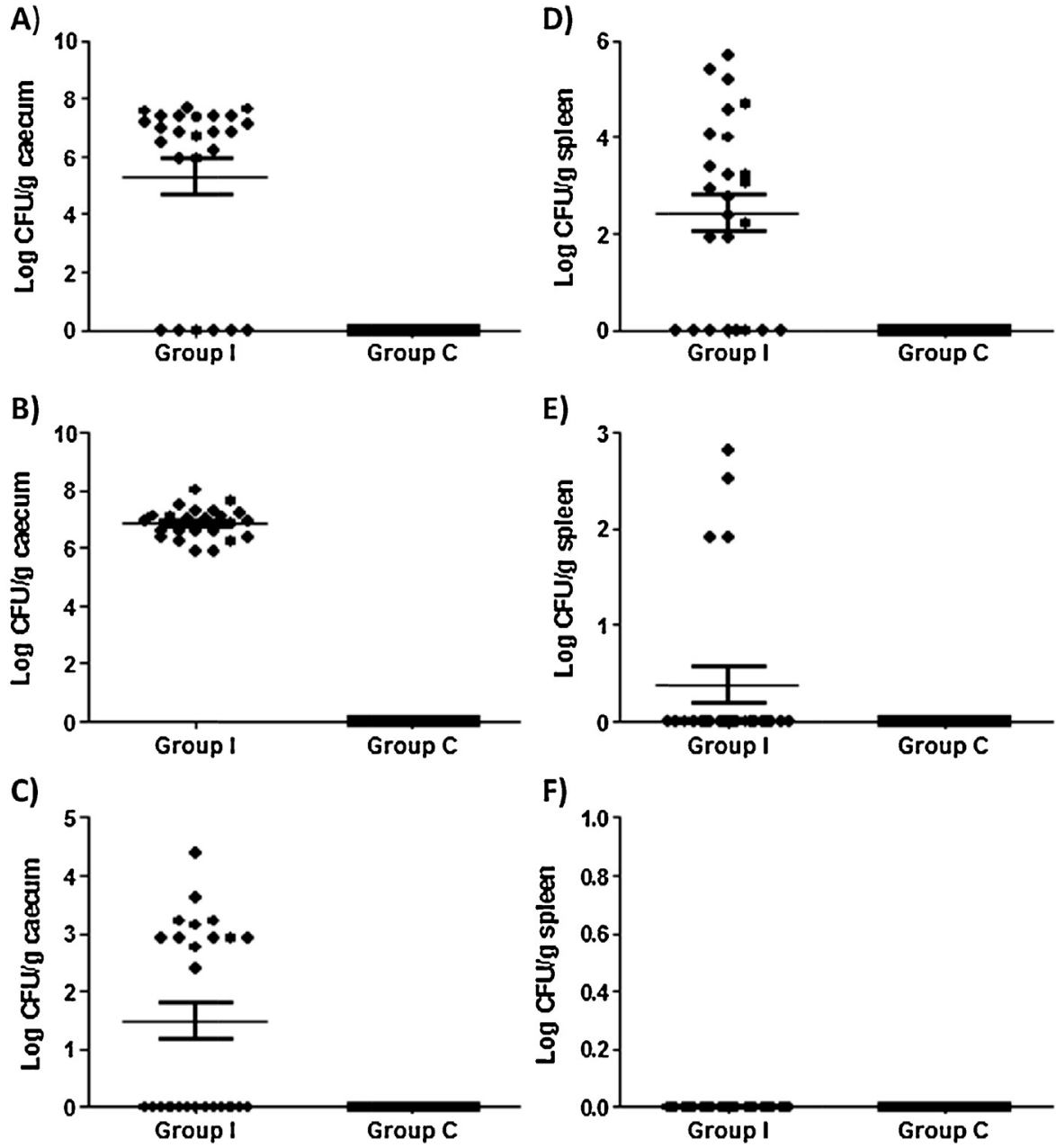

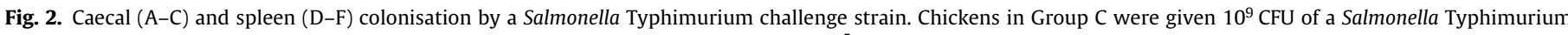

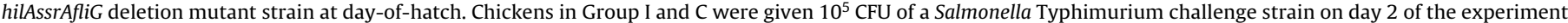

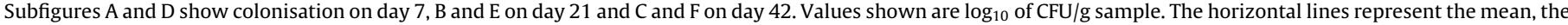

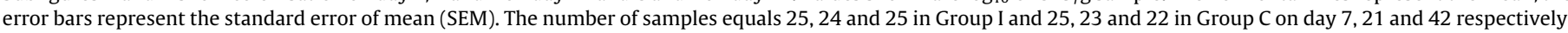

to similar high levels in Group V and Group C on day 7 (Fig. 1). The bacterial load of this strain was mean $\log _{10}=7.48 \mathrm{CFU} / \mathrm{g}$ in Group $\mathrm{V}$ and mean $\log _{10}=6.12 \mathrm{CFU} / \mathrm{g}$ in Group C. This reduced to mean $\log _{10}=6.01 \mathrm{CFU} / \mathrm{g}$ on day 21 and mean $\log _{10}=4.23 \mathrm{CFU} / \mathrm{g}$ on day 42 in Group V. The strain could no longer be detected on day 42 in Group C. No data were available for the caecum of Group C on day 21 due to overgrowth of other bacteria on the culture media. In the spleen, the bacterial load of the Salmonella Typhimurium $\Delta$ hilAssrAfliG strain amounted on day 7 to mean $\log _{10}=2.05 \mathrm{CFU} / \mathrm{g}$ in Group V and $\log _{10}=1.61 \mathrm{CFU} / \mathrm{g}$ in Group C. Bacterial numbers reduced as the experiment proceeded, as the load amounted to mean $\log _{10}=0.337 \mathrm{CFU} / \mathrm{g}$ in Group V and mean $\log _{10}=0.341 \mathrm{CFU} / \mathrm{g}$ in Group C on day 21. This reduced further to mean $\log _{10}=0.097$ $\mathrm{CFU} / \mathrm{g}$ in Group V on day 42. By then, the Salmonella Typhimurium $\Delta$ hilAssrAfliG strain could no longer be detected in Group C.

Bacteriological analysis of the caecum and spleen showed that the Salmonella Typhimurium challenge strain colonised the caecum of the chickens in Group I to high levels, while it could not be detected in any of the caeca of the chickens in Group $C$ at any time point (Fig. 2). The bacterial load and number of spleens positive for the Salmonella Typhimurium challenge strain in Group I was initially high, but declined as the experiment proceeded. The Salmonella Typhimurium challenge strain could not be detected in any of the spleens on day 42 in Group I, and in any of the spleens of the chickens belonging to Group $\mathrm{C}$ at any time point.
4.2. Experiment 2: efficacy of simultaneous administration of a Salmonella Typhimurium hilAssrAfliG and a Salmonella Enteritidis hilAssrAfliG deletion mutant against infection with several Salmonella serotypes

None of the chickens died during the experiment. Bacteriological analysis of the samples showed that the Salmonella Enteritidis and Typhimurium $\triangle$ hilAssrAfliG strains colonised the caecumand spleen to a similar level in all treated groups. Mean colonisation was $\log _{10}=6.87 \pm 0.12$ and $6.44 \pm 0.76 \mathrm{CFU} / \mathrm{g}$ in the caeca, and $\log _{10}=0.74 \pm 0.50$ and $2.02 \pm 0.77 \mathrm{CFU} / \mathrm{g}$ in the spleens of Groups VSE and VST, respectively. Data on colonisation by the CI culture in Group VSJ is not available because the Salmonella Paratyphi B var. Java strain is, like the CI strains, nalidixic acid resistant. Consequently, the $\mathrm{CI}$ strains could not be distinguished from the challenge strain. Additionally, bacteriological analysis of caecum and spleen showed that colonisation by the Salmonella Enteritidis challenge strain was significantly lower in the caecum of the group treated with CI culture (Fig. 3). No differences could be observed in spleen colonisation. Similarly, colonisation by the Salmonella Typhimurium strain was significantly lower in the caecum of the treated group, while no significant difference could be observed in the spleen. Colonisation by the Salmonella Paratyphi B var. Java strain did not differ significantly between the treated and untreated group. 
A)

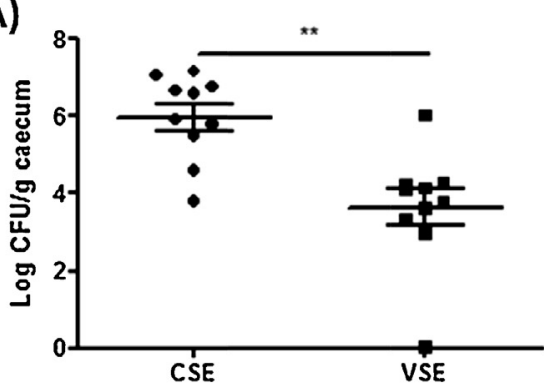

B)

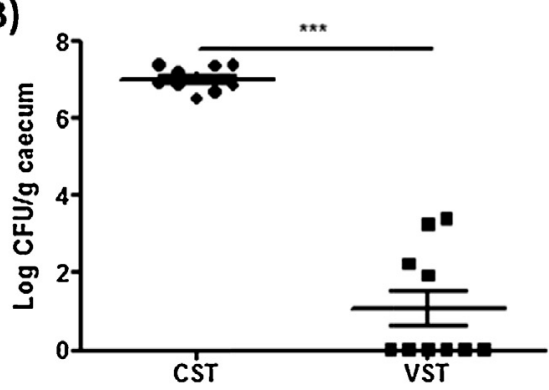

C)

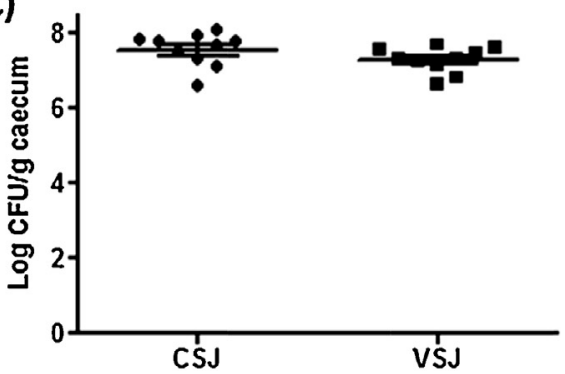

D)

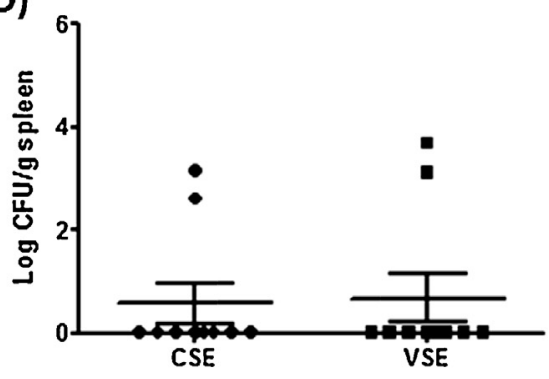

E)

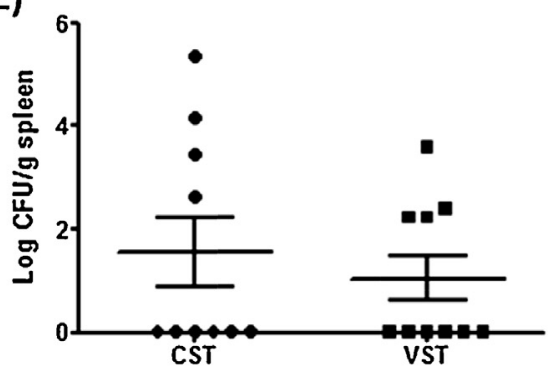

F)

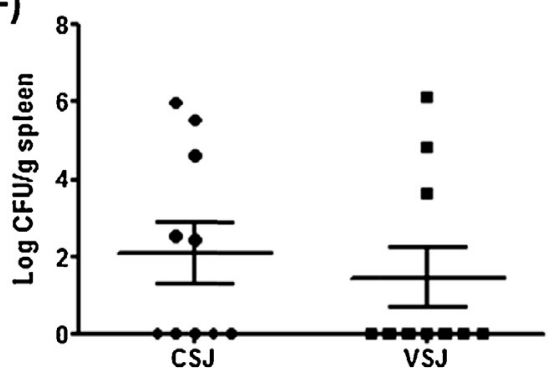

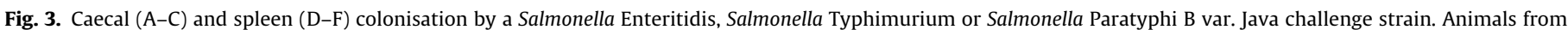

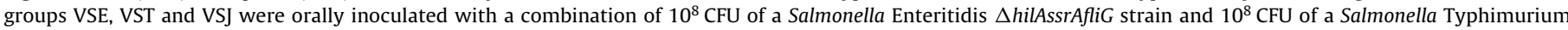

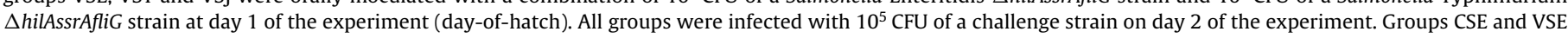

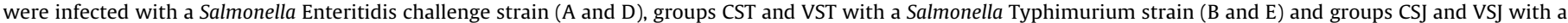
Salmonella Paratyphi B var. Java strain (C and F). Samples were taken at day 7 of the experiment.

\section{Discussion}

Newly hatched chicks are highly susceptible to Salmonella infections during the first days of life and inoculation with very low doses can result in persistent infections $[2,8,9]$. This high susceptibility has been associated with the absence of normal gut microbiota $[3,4]$ and the immature immune system of young chickens [5-7]. As a consequence, classical vaccination is not an effective means to achieve protection against Salmonella infection during the first days of life [14]. Alternatively, the use of organic acids, essential oils, proand prebiotics as feed supplements can help to control Salmonella infections in broiler chickens, but the protective responses elicited by these compounds only start several days post-hatch [22-24]. Since a rapid colonisation-inhibiting effect has been described in birds inoculated with a live Salmonella strain that protected the animals against subsequent challenge with another Salmonella strain, administration of $\mathrm{CI}$ strains to chickens early post-hatch might be a valuable addition to these strategies.

The Salmonella Typhimurium $\Delta$ hilAssrAfliG strain used in the present study was very effective at protecting against Salmonella Typhimurium challenge. Unfortunately, the Salmonella Typhimurium $\Delta$ hilAssrAfliG strain was highly colonising and persisted in the caecum until slaughter age when the chickens were not challenged with a wild-type strain. However, when challenging the chickens with a wild-type strain, the Salmonella Typhimurium $\Delta$ hilAssrAfliG strain was cleared rapidly from the chickens. This suggests an interaction between both strains that influences persistence and clearance of the $\mathrm{CI}$ strain from the chickens. Still, because this might result in the introduction of the deletion mutant strain in the food chain when applied in the field, the developed CI strain might not be an appropriate candidate for use in broiler production. As the ability of Salmonella strains to colonise represents an important prerequisite for effective colonisation inhibition of wildtype strains, persistence of a CI strain and protection offered by a CI strain are probably related to each other. Furthermore, it has been suggested that a $\mathrm{CI}$ strain is more protective against challenge when it is highly colonising [16,25]. It is therefore not improbable that the observed strong protective effect of the Salmonella Typhimurium $\triangle$ hilAssrAfliG strain is due to its high colonising capacity. A Salmonella Enteritidis hilAssrAfliG deletion mutant has been shown to colonise spleen and caecum to a lesser extent and was cleared rapidly from poultry, but also offered relatively less protection against Salmonella Enteritidis infection [15]. These and earlier observations suggest thus that there will be a trade-off between persistence and protection, as a highly colonising and thus protective strain will probably not be eliminated by slaughter age 
[26]. In contrast, a strain that is poorly colonising will be eliminated by slaughter age, but will probably not offer a long lasting protection. Obviously, these aspects should be taken into account when developing a $\mathrm{CI}$ strain.

Earlier research showed that the colonisation-inhibition effect is more pronounced between isogenic strains and that there is greater inhibition within a serovar than between serovars [14,16,27]. Consequently, it is likely that the Salmonella Typhimurium $\Delta$ hilAssrafliG strain is not able to inhibit strains belonging to other serovars than Typhimurium. It has however been suggested that a mixture of Salmonella strains belonging to several serovars would be able to inhibit a broad spectrum of virulent wild-type strains [16]. Therefore, we investigated the protective properties of a mixed culture consisting of both the Salmonella Enteritidis and Typhimurium $\Delta$ hilAssrAfliG strain against infection by 3 different Salmonella serovars. The results obtained in this study showed that the combined $\mathrm{CI}$ culture confers protection against a non-isogenic Salmonella Enteritidis and Salmonella Typhimurium challenge strain quickly after hatching. This suggests that both CI strains do not inhibit each other, or if they do it is to such a limited extent that they do not impede each other's protective properties. Earlier research showed that administering a mixture consisting of a wild-type Salmonella Enteritidis and Salmonella Typhimurium strain resulted in a pronounced protection against their isogenic challenge strains, but also against Salmonella Hadar and Salmonella Infantis challenge strains [16]. This suggests a synergistic protective effect when administering multiple CI strains simultaneously. In the present study, the CI mixture consisting of the Salmonella Enteritidis $\Delta$ hilAssrAfliG strain and Salmonella Typhimurium $\Delta$ hilAssrAfliG strain did, however, not offer protection against challenge with a Salmonella Paratyphi B. var.Java strain. Consequently, this suggests that a Salmonella Paratyphi B. var. Java $\mathrm{CI}$ strain needs to be developed and added to the CI culture in order to achieve simultaneous protection against Salmonella Enteritidis, Typhimurium and Paratyphi B. var. Java infection.

In conclusion, a significant reduction in faecal shedding and caecal and internal organ colonisation by a virulent Salmonella Typhimurium challenge strain could be obtained by administering a Salmonella Typhimurium $\Delta$ hilAssrAfliG mutant strain to one day old broiler chickens. Additionally, when this strain was administered simultaneously with a Salmonella Enteritidis hilAssrAfliG deletion mutant protection against infection by both a Salmonella Enteritidis and Typhimurium challenge strain could be obtained. These data demonstrate that colonisation-inhibition represents a promising tool to protect broilers early after hatching against multiple Salmonella serotypes. They pave the way for developing new $\mathrm{CI}$ strains and CI cultures that are cleared at slaughter age and protect against a wide variety of Salmonella serovars that are of importance for broiler production.

\section{Acknowledgements}

We would like to thank the many PhD students of the department of Pathology, Bacteriology and Avian Diseases for their skilled technical assistance. This work was funded by grant RF 09/6221 of the Belgian Federal Service for Public Health, Food Chain Safety and Environment.

\section{References}

[1] EFSA. The European Union summary report on trends and sources of zoonoses, zoonotic agents and food-borne outbreaks in 2011. EFSA J 2013;11:19-73.

[2] Desmidt M, Ducatelle R, Haesebrouck F. Pathogenesis of Salmonella Enteritidis phage type four after experimental infection of young chickens. Vet Microbiol 1997;56:99-109.
[3] Crhanova M, Hradecka H, Faldynova M, Matulova M, Havlickova H, Sisak $\mathrm{F}$, et al. Immune response of chicken gut to natural colonization by gut microflora and to Salmonella enterica serovar Enteritidis infection. Infect Immun 2011;79:2755-63.

[4] Coloe PJ, Bagust TJ, Ireland L. Development of the normal gastrointestinal microflora of specific pathogen-free chickens. J Hyg (Lond) 1984;92:79-87.

[5] Vainio O, Imhof BA. The immunology and developmental biology of the chicken. Immunol Today 1995;16:365-70.

[6] Bar-Shira E, Sklan D, Friedman A. Establishment of immune competence in the avian GALT during the immediate post-hatch period. Dev Comp Immunol 2003;27:147-57.

[7] Bar-Shira E, Friedman A. Development and adaptations of innate immunity in the gastrointestinal tract of the newly hatched chick. Dev Comp Immunol 2006;30:930-41.

[8] Van Immerseel F, De Buck J, Pasmans F, Bohez L, Boyen F, Haesebrouck F, et al. Intermittent long-term shedding and induction of carrier birds after infection of chickens early posthatch with a low or high dose of Salmonella Enteritidis. Poult Sci 2004;83:1911-6.

[9] Gast RK, Benson ST. The comparative virulence for chicks of Salmonella Enteritidis phage type 4 isolates and isolates of phage types commonly found in poultry in the United States. Avian Dis 1995;39:567-74.

[10] Heyndrickx M, Vandekerchove D, Herman L, Rollier I, Grijspeerdt K, De Zutter L. Routes for Salmonella contamination of poultry meat: epidemiological study from hatchery to slaughterhouse. Epidemiol Infect 2002;129: 253-65.

[11] Barrow PA, Tucker JF. Inhibition of colonization of the chicken caecum with Salmonella Typhimurium by pre-treatment with strains of Escherichia coli. J Hyg (Lond) 1986;96:161-9.

[12] Bohez L, Dewulf J, Ducatelle R, Pasmans F, Haesebrouck F, Van Immerseel F. The effect of oral administration of a homologous hilA mutant strain on the long-term colonization and transmission of Salmonella Enteritidis in broiler chickens. Vaccine 2008;26:372-8.

[13] Barrow PA, Tucker JF, Simpson JM. Inhibition of colonization of the chicken alimentary tract with Salmonella Typhimurium gram-negative facultatively anaerobic bacteria. Epidemiol Infect 1987;98:311-22.

[14] Van Immerseel F, Methner U, Rychlik I, Nagy B, Velge P, Martin G, et al. Vaccination and early protection against non-host-specific Salmonella serotypes in poultry: exploitation of innate immunity and microbial activity. Epidemiol Infect 2005:133:959-78.

[15] De Cort W, Geeraerts S, Balan V, Elroy M, Haesebrouck F, Ducatelle R, et al. A Salmonella Enteritidis hilAssrAfliG deletion mutant is a safe live vaccine strain that confers protection against colonization by Salmonella Enteritidis in broilers. Vaccine 2013;31:5104-10

[16] Methner U, Haase A, Berndt A, Martin G, Nagy B, Barrow PA. Exploitation of intestinal colonization-inhibition between Salmonella organisms for live vaccines in poultry: potential and limitations. Zoonoses Public Health 2011;58:540-8

[17] Van Parys A, Boyen F, Verbrugghe E, Leyman B, Bram F, Haesebrouck F, et al. Salmonella Typhimurium induces SPI-1 and SPI-2 regulated and strain dependent downregulation of MHC II expression on porcine alveolar macrophages. Vet Res 2012;43:52.

[18] Datsenko KA, Wanner BL. One-step inactivation of chromosomal genes in Escherichia coli $\mathrm{K}-12$ using PCR products. Proc Natl Acad Sci USA 2000;97:6640-5.

[19] Van Immerseel F, De Buck J, De Smet I, Mast J, Haesebrouck F, Ducatelle R. Dynamics of immune cell infiltration in the caecal lamina propria of chickens after neonatal infection with a Salmonella Enteritidis strain. Dev Comp Immunol 2002;26:355-64.

[20] Methner U, al-Shabibi S, Meyer H. Infection model for hatching chicks infected with Salmonella Enteritidis. Zentralbl Veterinarmed B 1995;42:471-80.

[21] Methner U, al-Shabibi S, Meyer H. Experimental oral infection of specific pathogen-free laying hens and cocks with Salmonella Enteritidis strains. Zentralbl Veterinarmed B 1995;42:459-69.

[22] Van Immerseel F, Fievez V, de Buck J, Pasmans F, Martel A, Haesebrouck F, et al. Microencapsulated short-chain fatty acids in feed modify colonization and invasion early after infection with Salmonella Enteritidis in young chickens. Poult Sci 2004:83:69-74.

[23] Van Immerseel F, Boyen F, Gantois I, Timbermont L, Bohez L, Pasmans F, et al Supplementation of coated butyric acid in the feed reduces colonization and shedding of Salmonella in poultry. Poult Sci 2005;84:1851-6.

[24] Van Immerseel F, Russell JB, Flythe MD, Gantois I, Timbermont L, Pasmans F, et al. The use of organic acids to combat Salmonella in poultry: a mechanistic explanation of the efficacy. Avian Pathol 2006;35:182-8.

[25] Berchieri Jr A, Barrow PA. Further studies on the inhibition of colonization of the chicken alimentary tract with Salmonella Typhimurium by pre-colonization with an avirulent mutant. Epidemiol Infect 1990;104:427-41.

[26] Bohez L, Ducatelle R, Pasmans F, Haesebrouck F, Van Immerseel F. Long-term colonisation-inhibition studies to protect broilers against colonisation with Salmonella Enteritidis, using Salmonella Pathogenicity Island 1 and 2 mutants. Vaccine 2007;25:4235-43.

[27] Methner U, Barrow PA, Gregorova D, Rychlik I. Intestinal colonisationinhibition and virulence of SalmonellaphoP, rpos and ompC deletion mutants in chickens. Vet Microbiol 2004;98:37-43. 